\title{
Coal Refinery Process Absorbability Index Assessment against Foot Print of Air Pollution by Usage of Robust Optimization Algorithms: A Novel Green Environment Initiative
}

\author{
Hua Xu $\mathbb{D}^{1},{ }^{1}$ Shuqiang Cheng, ${ }^{1}$ M. Prabhu $\mathbb{D}^{2}$, and Anoop Kumar Sahu $\mathbb{D}^{3}$ \\ ${ }^{1}$ School of Economics and Management, Shaanxi University of Science and Technology, Xi'an Shaanxi 710021, China \\ ${ }^{2}$ Assistant Professor, Department of Business Administration, Lebanese French University, 100 MTR Avenue, \\ 44001 Erbil Kurdistan Region, Iraq \\ ${ }^{3}$ Faculty, Mechanical Engg, Guru Ghasidas Vishwavidyalaya, Bilaspur, C.G, India
}

Correspondence should be addressed to Hua Xu; xuhua0910@sina.cn

Received 1 June 2021; Accepted 8 September 2021; Published 18 November 2021

Academic Editor: Shien Hui

Copyright (C) 2021 Hua Xu et al. This is an open access article distributed under the Creative Commons Attribution License, which permits unrestricted use, distribution, and reproduction in any medium, provided the original work is properly cited.

Coals are employed as fiery substance, and every day, millions of ton coal are consumed by coal users around the world. It is investigated that the millions of coal's transportation/logistic till the coal user plants via road route and also inside the coal user plants (known as twice factors) not only enhance the air pollution but also cause the global warming. It is earlier known that coals emit the toxic pollutants and offensive gases such as sulfur dioxide, $\mathrm{SO}_{2}$; nitrogen oxides, NOX; hydrogen chloride arsenic; carbon monoxide, $\mathrm{CO}$; methane; $\mathrm{CH}_{4}$; and $\mathrm{CO}_{2}$ on reacting with environmental $\mathrm{O}_{2}$ due to said twice factors, i.e., during the transportation from coal refinery spot to entry gate of coal user plants (another spot) and in process logistic/movement inside the coal user plants (loading to conveyor to coal fire tubes "attached with coal crushers"). Therefore, the coal refinery technique/process is found as the best practice to control air pollution under concerns of twice factors. The reliable and trustworthy coal refining technology improves the quality of coal by eradicating or eliminating the coating or layers of toxic particles from coal's surface, which speedily crumble or decompose in reacting with environmental $\mathrm{O}_{2}$ under twice factors. As results, coal refining technology adds the green supply chain value into proposed twice factors and also save the world from breeding of ills and viruses. It is understood that the best coal refinery technique/process helps to overcome and reduce air pollution by responding discussed twice factors (accepted as research challenge and motivation of research). In the presented research work, the authors developed and proposed a dynamic multidimension Coal Refinery Process Absorbability Index (CRPAI) structure (consisted of coal refinery core dimension and subdimensions correspond to CRPA alternative techniques/processes) appended with Robust Optimization Algorithm (ROA) to be explored for opting the best CRP from available options. But due to inherent ambiguity, vagueness, and inconsistency involve in both dimensions of proposed structure, the assessment of expert's panel is gathered in the terms of linguistic variable "appropriateness ratings" against the subdimensions of CRPAI structure corresponding to preferred CRP options. Next, assigned appropriateness ratings against the subdimensions are substituted by GIVFN. To arrive to core dimensions from subdimensions of CRPAI structure, a GITFN-OWGO (Ordered Weighted Geometric Operator) is investigated and modified as a Ordered Weighted Geometric Average Operator (OWGAO) to be applied for estimating the weights of subdimensions (core novelty of work). Finally, a ROA (consisted of MULTI-MOORA with dominance theory) is applied on the output of OWGAO for opting the viable and best CRP option. The positive effect of the dynamic multidimension CRPAI structure is that it helps the coal refinery companies to assess measure and evaluate the best and feasible coal refinery process under concern of twice factors using expert information. The research can be used to control the air pollution by responding aforesaid twice factors by single practice (the best coal refinery process/technique assessment and evaluation). 


\section{Introduction and State of Art}

The $\mathrm{CO}_{2}$ is ascertained as the foremost carbon intensive, which is accountable for more than $72 \%$ climate altering phenomenon and major source of air pollution and global warming vice versa. Today, coals are employed as burning material across the global coal user plants. The coal is found chemically intricate fuel as it librates flue gases, which might contain such as sulfur dioxide, $\mathrm{SO}_{2}$; nitrogen oxides, $\mathrm{NOX}$; hydrogen chloride arsenic; carbon monoxide, $\mathrm{CO}$; methane; $\mathrm{CH}_{4}$; and carbon dioxide, $\mathrm{CO}_{2}$ [1-10]. Every day, millions of ton coal are fired by coal user plants. It is noticed that coal becomes extremely low contaminated/toxic gas emitter from coal refinery location/spot to entry gate of coal user plants and also throughout the logistic of coal inside the coal user plants if coal refinery is carried with best practices and technique at inception. Therefore, coal refinery is searched as the preeminent practice amongst many green practices to clean the environment and control air pollution. The coal refinery is respected as ecological balancer in the world, which function is to refine the coal to eliminate further air pollution ([6, 11-15]; CURC 2004; [1-5, 7-10, 16, 17]).

Refined coals are the burnable goods, which are produced by application of a coal-upgrading practices-technology, which eliminate the moisture and certain pollutants from surface of coal stone and add the calorific values. Coal refining is precombustion treatments or processes, which changes the characteristics of coal prior to move is to be burned. In India nation, the coal refinery company use, such as dynamic process, is exhibited here: crushing and breaking-crushing and breaking devices are feeder breakers, rotary breakers, hammer mills, and roll crushers. Sizing the$\mathrm{m}$-it is sizing the coal under 20 to $45 \mathrm{~mm}$ diameters. Storage and stockpiling-coal is stored in silos or stockpiled before and after cleaning. Spherical, cylindrical, conical silos, or stockpiled is used. Density separation-coal is cleaned by separating the lower density organic material from the higher density by falling it from high 7-10 m height to earth. Washing-washing is done by quenching algorithm and passing through flowing water. Coal drying - thermal drying is lastly carried out by using container, drying in open overcast by sun to reduce the moisture content.

In investigating the CRPAI research documents (RDs), the authors found the many RDs in relation to analyzing the coal supplier performance and materializing the coal emission quantities around the coal user plants. So few RDs are found, dealt with mapping CRPAI under core dimension modeling by certain or quantitative data (except core dimension modeling by uncertain or qualitative data). In addition to that, few CRPA index documents are also grasped, dealt with CRPAI, but constrained to consider the core dimension (except both: core and subdimension simultaneously) [2-4, 7, 9, 16].

Furthermore, in investigating the RDs against ROAs, the authors determined that most of the Absorbability Index evaluation RDs dealt with the single optimization algorithm (could tackle certain data vs. core dimension only). The authors were not found still relevant research document, especially dealt with dynamic multidimension CRPAI struc- ture (consisted of coal refinery core dimension and subdimensions correspond to CRPA alternative processes) with ROAs (can tackle uncertain/qualitative data vs. both: core and subdimension only) to be used for opting the best CRP from available options. Therefore, the aforesaid research gaps motivated the authors to develop and propose a dynamic multidimension CRPAI structure (consisted of coal refinery core dimension and subdimensions) with ROA to opt the best CRP from available options under linguistic information of expert's panel.

To construct a dynamic multidimension CRPAI structure in addressing the qualitative modeling of core and subdimension simultaneously (CRPAI structure) and finding ROAs, the authors conduct a few literature surveys under aforesaid concerns:

\subsection{A Literature over Framing the Dynamic Multidimension} CRPAI Structure. [18-21], and [22] outlined the nature of the wastes; waste generating industries; waste characterization; health and environmental implications of waste management practices; steps towards planning; design and development of models for effective hazardous waste management; and treatment, approaches, and regulations for disposal of hazardous waste. Evaluation of the entire situation with reference to Indian scenario has been attempted in order that a better cost-effective strategy for waste management be evolved in the future. [23] proposed a decision model based on environmental performance indicators, which supported decision-making in supply chains in presence of environmental considerations. [24] implemented IVFN-TOPSIS (interval-valued fuzzy number-Technique for Order Preference Similar to Ideal Solution) approach in purpose to find out preference orders of manufacturing alternative firms in context of green supply chain management. [25] articulated an innovative, quantitative assessment of pollution avoidance attributable to environmental regulation enforced through integrated licensing, using Ireland's pharmaceutical-manufacturing sector as a case study. [16] argued the current coal consumption in the UK united with ecological impacts of coal mining, coal processing, and coal utilization for power generation. [7] shown impact of coal mining on land, water, health of workers, air, and social impact on surrounding villages and cities have evaluated. In addition to that, environment management plan of coal mining has also enlarged. [9] carried out a coal mining project in northwestern Bangladesh country in order to appraise the impacts of coal mining on the surrounding environment specifically on soil and water. The research of [26] was conducted to seek the miscellaneous paths to preserve the environmental and also put the environment out of fewer emission of carbon dioxide. The countless several conducted state of arts have been depicted as a citation in order that readers could seek the other way to diminish the universal warming issues in planet world ([1, 2, 8, 10, 17, 27-29]; Sahu et al., 2012; Sahu et al., 2013; [7, 30]a, b; [9, 22]).

Kalibatas and Turskis 2008 and [26] have also formed miscellaneous decision support system in context of manufacturing realm. [31] thermally treated the petroleum coke in the range of $1173-1673 \mathrm{~K}$. The changes of the 
composition and crystalline structure of petroleum coke were investigated. $([11,14,15] ;$; $[1-10,13,16,17,24,30]$ a, b) worked to reduce air pollution by applying multivariable optimization methods. Venugopal et al. [32] stimulate the need for developing native solutions against the industrial challenges and revealed the importance of coal industry and coal washing process. Dhawan et al. [33] conducted an experiments for investigating the process parameters of catalytic coke pretreatment processes parameters. Sitorus et al. [34] applied a hybrid MCDM method to evaluate the coal mining and mineral processing units and discussed the pathways and future research directions of the proposed MCDM method. Pourabdollah et al. [35] studied the effect of thermal operation parameters against the graphitization of ethane-based catalytic coal. Dhawan et al. [36] investigated the usage of the catalyst $\mathrm{K}_{2} \mathrm{CO}_{3}$ over raw coal and the use of $\mathrm{KCO}_{3}$ over the raw coal as well as help to extract the $\mathrm{CO}_{2}$ gas from raw coal. Shukla et al. [37] studied about the emission level of $\mathrm{CO}_{2}$ in India from coal. Various advanced methods for capturing $\mathrm{CO}_{2}$ are highlighted.

1.2. Literature over Framing the ROAs. [38] explored the application of multiobjective optimization on the basis of ratio analysis (MOORA) algorithm to solve different decision-making problems as frequently encountered in the real-time manufacturing environment. Six decision-making problems have been solved to prove the applicability, potentiality, and flexibility of this algorithm while solving various complex decision-making problems in present day manufacturing environment. [39] implemented the MOORA algorithm for solving multiple criteria (objective) optimization problem in milling process. Six decisionmaking problems which include selection of suitable milling process parameters in different milling processes are considered, and the obtained results almost match with those derived by the previous researchers which prove the applicability, potentiality, and flexibility of this algorithm in manufacturing environment subsequently. Sahu et al. 2012, Sahu et al. 2013, [30] a, b, [9, 22] have conducted research in context of environmental subdimensions, which have been viewed as a case study. Sahu et al. [24] applied a weighted geometric aggregation operator on robot evaluation model to score the industrial value of robots. Bu et al. [40] proposed a GWmZd model consisted of green vendor evaluation parameters and solved it by knowledge-based grey-holistic technique with dominance theory for sustainable vendor evaluation. He et al. [41] proposed an advanced manufacturing machine tool evaluation model under objective information and solved it by grey relational analysis approach with dominance theory.

1.3. Summary of State of Art. As discussed, the authors only highlighted so few relevant literatures with brief description and cited more RDs to shorten research work. After literature survey, the authors consolidated the relevant CRPAI core and subdissensions and framed a dynamic multidimension CRPAI structure (consisted of coal refinery core dimension and subdimensions). In addition to that, the authors found a most significant ROA (MULTI-MOORA) and enable same to opt the best CRP from available options under linguistic information of expert's panel. But searched ROA was able for qualitative modeling of core dissensions. By using concept of fuzzy average rule, the authors made ROA able to tackle core and subdissensions simultaneously by introducing modification in the OWG operator (in previous researches, operator was able to act for qualitative modeling of only core level dimension). Moreover, on focusing over the most recent research of $\mathrm{Bu}$ et al. [40] and $\mathrm{He}$ et al. [41] for obtaining the reliable results, the authors applied the dominance theory for the comparison amongst the CRPAI preferences, provided by ROA.

\section{Rationale to Conduct the Research}

Coal pollutants mixed into the atmosphere, which disorder the ecosystem, are shown in Figure $1([38,39,42-47]$; Sahu et al., 2012; Sahu et al., 2013; [30] a, b; [9, 22]).

As discussed, twice factors (transportation from coal refinery spot to entry gate of coal user plants (another spot) and in process logistic/movement inside the coal user plants (loading to conveyor to coal fire tubes attached with coal crushers)) push the attention of authors to conduct the research work in the context of CRP. The authors found a preresearch clue to overcome and control the air pollution and global warming vice versa ([25, 48, 49]; Sahu et al., 2012; Sahu et al., 2013; [30] a, b; [22]).

Later, the authors preaudited the RDs of CRPAI and ascertained that there are still no RDs pertaining to CRPAI structure, which can deal with the qualitative modeling of both core and subdimensions. Up next, the authors found that there are a few RDs related to evaluation of CRPAI by executing single optimization algorithms (SOAs) with the OWG operator (operator was able to act for qualitative modeling of only core level dimension). The authors claimed that there is no RD yet, which can tackle subdimensions of CRPAI structure under qualitative modeling in application of the OWG operator appended with ROAs $([30,50-52] c$, e).

Therefore, the aforesaid rationale stimulated the authors to develop and propose a dynamic multidimension CRPAI structure (consisted of coal refinery core dimension and subdimensions) with constructing ROAs (MULTI-MOORA with modification of OWG operator by average fuzzy rule) with application of dominance theory ( $\mathrm{Bu}$ et al., [40] and He et al., [41] for evaluation of the best CRP from available options under the linguistic information of expert's panel. This proposed research work would assist the managers to identify the best CRP practice and technique or to opt viable CRPs amongst preferred CRPs.

\section{Rationale to Implement Interval-Valued Fuzzy Number Set (IVFNs) than Other Fuzzy Number Set}

In the present context, authors fruitfully implemented IVFN except other fuzzy numbers, as IVFN deals with completely unknown information. In IVFNs, fuzzy membership function values are intended in between 0 and 1 , aligned over 


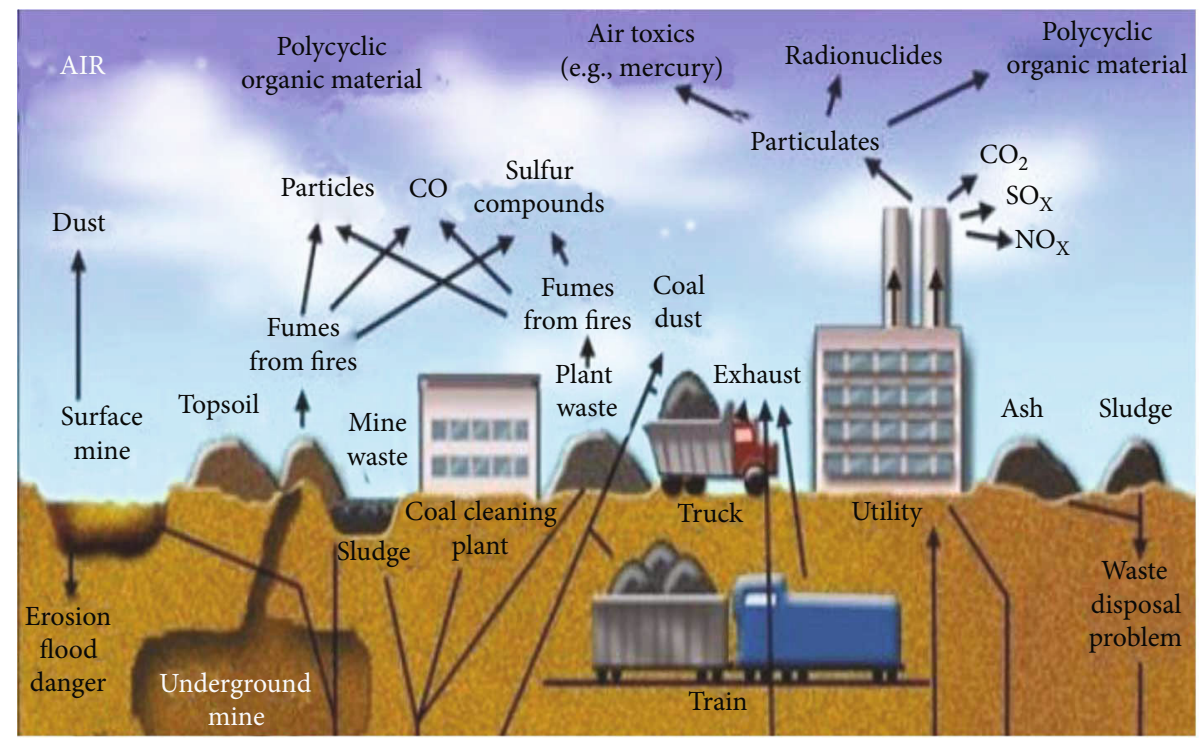

Figure 1: Disordering of ecosystem from coal under twice factors.

two boundaries (upper level $\left(U^{U}\right)$ and lower level $\left(U^{L}\right)$ of membership function. The considered boundaries also undertake the space between $\left(U^{u}\right)$ and $\left(U^{L}\right)$ and can tackle the imprecise, haziness, and vagueness associated with information of expert panel against defined core-subdimensions $([46,50,51,53,54]$; Sahu et al., 2013; [30]), while other fuzzy numbers only deal with the single boundary, which not at all prove to engrave the imprecise, haziness, and vagueness associated with information of expert panel. Consequently, due to aforementioned rationale, IVFN is used for qualitative modeling of defined both dimensions (expert panel's information) for making the verdict $[52,55-59]$.

\section{Fuzzy Preliminaries}

Fuzzy sets and fuzzy logic are powerful mathematical tools, employed for modeling uncertain systems or qualitative information. A fuzzy set is an extension of a crisp set. A crisp set only allows full membership or nonmembership, while fuzzy sets allow the partial membership. The theoretical fundaments of fuzzy set theory are overviewed by $[52,55,57-61]$. This section presents the generalized interval-valued trapezoidal fuzzy numbers. In addition, the arithmetic operations and aggregation of the generalized interval-valued trapezoidal fuzzy numbers are as follows.

4.1. The Generalized Trapezoidal Fuzzy Numbers. A fuzzy set $\tilde{A}$ in a universe of discourse $X$ is characterized by a membership function $\mu_{\tilde{A}}(x)$ which associates with each element $x$ in $X$ a real number in the interval $[0,1]$. The function value $\mu_{\tilde{A}}(x)$ is termed the grade of membership of $x$ in $\tilde{A}$. A trapezoidal fuzzy number can be defined as $\tilde{A}=\left(a_{1}, a_{2}, a_{3}, a_{4}\right.$; $\left.w_{\tilde{A}}\right)$ as shown in Figure 2, and the membership function
$\mu_{\tilde{A}}(x): R \longrightarrow[0,1]$ is defined as follows:

$$
\mu_{\tilde{A}}(x)= \begin{cases}\frac{x-a_{1}}{a_{2}-a_{1}} \times w_{\tilde{A}}, & x \in\left(a_{1}, a_{2}\right), \\ w_{\tilde{A}}, & x \in\left(a_{2}, a_{3}\right), \\ \frac{x-a_{4}}{a_{3}-a_{4}} \times w_{\tilde{A}}, & x \in\left(a_{3}, a_{4}\right), \\ 0, & x \in\left(-\infty, a_{1}\right) \cup\left(a_{4}, \infty\right) .\end{cases}
$$

Here, $a_{1} \leq a_{2} \leq a_{3} \leq a_{4}$ and $w_{\tilde{A}} \in(0,1)$.

Suppose that $\tilde{a}=\left(a_{1}, a_{2}, a_{3}, a_{4} ; w_{\tilde{A}}\right)$ and $\tilde{b}=\left(b_{1}, b_{2}, b_{3}\right.$, $\left.b_{4} ; w_{\tilde{B}}\right)$ are two trapezoidal fuzzy numbers, then the operational rules of the trapezoidal fuzzy numbers $\tilde{a}$ and $\tilde{b}$ are shown as follows:

$$
\begin{aligned}
\tilde{a} \oplus \tilde{b} & =\left(a_{1}, a_{2}, a_{3}, a_{4} ; w_{\tilde{A}}\right) \oplus\left(b_{1}, b_{2}, b_{3}, b_{4} ; w_{\tilde{B}}\right) \\
& =\left(a_{1}+b_{1}, a_{2}+b_{2}, a_{3}+b_{3}, a_{4}+b_{4} ; \min \left(w_{\tilde{A}}, w_{\tilde{B}}\right)\right), \\
\tilde{a}-\tilde{b} & =\left(a_{1}, a_{2}, a_{3}, a_{4} ; w_{\tilde{A}}\right)-\left(b_{1}, b_{2}, b_{3}, b_{4} ; w_{\tilde{B}}\right) \\
& =\left(a_{1}-b_{4}, a_{2}-b_{3}, a_{3}-b_{2}, a_{4}-b_{1} ; \min \left(w_{\tilde{A}}, w_{\tilde{B}}\right)\right),
\end{aligned}
$$

$$
\begin{aligned}
\tilde{a} \otimes \tilde{b} & =\left(a_{1}, a_{2}, a_{3}, a_{4} ; w_{\tilde{A}}\right) \otimes\left(b_{1}, b_{2}, b_{3}, b_{4} ; w_{\tilde{B}}\right)=\tilde{a} \otimes \tilde{b} \\
& =\left(a_{1} \times b_{1}, a_{2} \times b_{2}, a_{3} \times b_{3}, a_{4} \times b_{4} ; \min \left(w_{\tilde{A}}, w_{\tilde{B}}\right)\right),
\end{aligned}
$$

$\frac{\tilde{a}}{\tilde{b}}=\frac{\left(a_{1}, a_{2}, a_{3}, a_{4} ; w_{\tilde{A}}\right)}{\left(b_{1}, b_{2}, b_{3}, b_{4} ; w_{\tilde{B}}\right)}=\left(\frac{a_{1}}{b_{4}}, \frac{a_{2}}{b_{3}}, \frac{a_{3}}{b_{2}}, \frac{a_{4}}{b_{1}} ; \min \left(w_{\tilde{A}}, w_{\tilde{B}}\right)\right)$.

[27] introduced the center of gravity (COG) measure for generalized trapez4oidal fuzzy numbers. Let there be a generalized trapezoidal fuzzy number $\tilde{A}=\left(a_{1}, a_{2}, a_{3}, a_{4} ; w_{\tilde{A}}\right)$. 


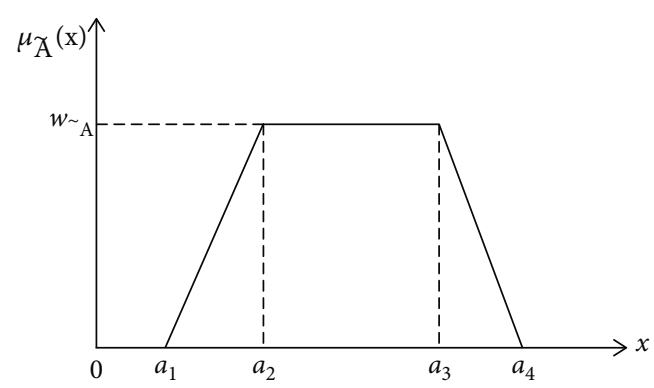

Figure 2: Trapezoidal fuzzy number $\tilde{A}$.

Then, it has its COG point $\left(x_{\tilde{A}}, y_{\tilde{A}}\right)$, where

$$
\left\{\begin{array}{l}
y_{\tilde{A}}=\left\{\begin{array}{l}
w_{\tilde{A}}\left(\frac{a_{3}-a_{2}}{a_{4}-a_{1}}+2\right), a_{1} \neq a_{4} \\
\frac{w_{\tilde{A}}}{2}, a_{1}=a_{4}
\end{array}\right. \\
x_{\tilde{A}}=\frac{y_{\tilde{A}}\left(a_{2}+a_{3}\right)+\left(a_{1}+a_{4}\right)+\left(w_{\tilde{A}}-y_{\tilde{A}}\right)}{2 w_{\tilde{A}}}
\end{array} .\right.
$$

4.2. The Generalized Interval-Valued Trapezoidal Fuzzy Numbers. Some basic concepts of IVFNs and their arithmetic operations are discussed below:

[57] defined IVFNs and presented their extended operational rules. The trapezoidal IVFN $\widetilde{\tilde{A}}$ has been represented by $([46,50,51,53,55]$; Sahu et al., 2013; [30]).

$$
\widetilde{\widetilde{A}}=\left[\widetilde{\tilde{A}}^{L}, \widetilde{\tilde{A}}^{U}\right]=\left[\left(a_{1}^{L}, a_{2}^{L}, a_{3}^{L}, a_{4}^{L} ; w_{\tilde{A}}^{L}\right),\left(a_{1}^{U}, a_{2}^{U}, a_{3}^{U}, a_{4}^{U} ; w_{\tilde{\tilde{A}}}^{U}\right)\right],
$$

Here, $a_{1}^{L} \leq a_{2}^{L} \leq a_{3}^{L} \leq a_{4}^{L}, a_{1}^{U} \leq a_{2}^{U} \leq a_{3}^{U} \leq a_{4}^{U}, \widetilde{\tilde{A}}^{L}$ denotes the lower IVFN, $\widetilde{\tilde{A}}^{U}$ denotes the upper IVFN, and $\tilde{\tilde{A}}^{L} \subset \widetilde{\tilde{A}}^{U}$.

Assume that there are two IVFNs $\widetilde{\widetilde{A}}$ and $\widetilde{\widetilde{B}}$, where

$$
\begin{aligned}
& \widetilde{\tilde{A}}=\left[\widetilde{\tilde{A}}^{L}, \tilde{\tilde{A}}^{U}\right]=\left[\left(a_{1}^{L}, a_{2}^{L}, a_{3}^{L}, a_{4}^{L} ; w_{\tilde{\tilde{A}}}^{L}\right),\left(a_{1}^{U}, a_{2}^{U}, a_{3}^{U}, a_{4}^{U} ; w_{\tilde{A}}^{U}\right)\right], \\
& \widetilde{\widetilde{B}}=\left[\widetilde{\widetilde{B}}^{L}, \widetilde{\tilde{B}}^{U}\right]=\left[\left(b_{1}^{L}, b_{2}^{L}, b_{3}^{L}, b_{4}^{L} ; w_{\tilde{B}}^{L}\right),\left(b_{1}^{U}, b_{2}^{U}, b_{3}^{U}, b_{4}^{U} ; w_{\tilde{B}}^{U}\right)\right] .
\end{aligned}
$$

$$
0 \leq w_{\tilde{A}}^{L} \leq w_{\tilde{A}}^{U} \leq 1, \widetilde{\tilde{A}}^{L} \subset \widetilde{\tilde{A}}^{U}, 0 \leq w_{\tilde{B}}^{L} \leq w_{\tilde{B}}^{U} \leq 1 \text {, and } \widetilde{\widetilde{B}}^{L} \subset \widetilde{\widetilde{B}}^{U} .
$$

From Figure 3, it can be concluded that interval-valued trapezoidal fuzzy number $\widetilde{\tilde{A}}$ consists of the lower values of interval-valued trapezoidal fuzzy number $\widetilde{\tilde{A}}^{L}$ and the upper values of interval-valued trapezoidal fuzzy number $\widetilde{\tilde{A}}^{U}$. The operation rules of interval-valued trapezoidal fuzzy numbers as given by [57] have been reproduced below.
Suppose that

$$
\widetilde{\tilde{A}}=\left[\tilde{\tilde{A}}^{L}, \widetilde{\tilde{A}}^{U}\right]=\left[\left(a_{1}^{L}, a_{2}^{L}, a_{3}^{L}, a_{4}^{L} ; w_{\tilde{\tilde{A}} L}\right),\left(a_{1}^{U}, a_{2}^{U}, a_{3}^{U}, a_{4}^{U} ; w_{\tilde{\tilde{A}}}\right)\right],
$$

and

$$
\widetilde{\widetilde{B}}=\left[\widetilde{\widetilde{B}}^{L}, \widetilde{\widetilde{B}}^{U}\right]=\left[\left(b_{1}^{L}, b_{2}^{L}, b_{3}^{L}, b_{4}^{L} ; w_{\tilde{B}}\right),\left(b_{1}^{U}, b_{2}^{U}, b_{3}^{U}, b_{4}^{U} ; w_{\tilde{B}}^{U}\right)\right],
$$

are the two interval-valued trapezoidal fuzzy numbers, where

$$
\begin{gathered}
0 \leq a_{1}^{L} \leq a_{2}^{L} \leq a_{3}^{L} \leq a_{4}^{L} \leq 1, \\
0 \leq a_{1}^{U} \leq a_{2}^{U} \leq a_{3}^{U} \leq a_{4}^{U} \leq 1, \\
0 \leq w_{\tilde{A} L} \leq w_{\tilde{\tilde{A}}}^{U} \leq 1, \widetilde{\tilde{A}}^{L} \subset \widetilde{\tilde{A}}^{U}, \\
0 \leq b_{1}^{L} \leq b_{2}^{L} \leq b_{3}^{L} \leq b_{4}^{L} \leq 1, \\
0 \leq b_{1}^{U} \leq b_{2}^{U} \leq b_{3}^{U} \leq b_{4}^{U} \leq 1, \\
0 \leq w_{\tilde{B}}^{w_{L}} \leq w_{\tilde{B}} \leq 1, \widetilde{\widetilde{B}}^{L} \subset \widetilde{\widetilde{B}}^{U} .
\end{gathered}
$$

(i) The sum of two interval-valued trapezoidal fuzzy numbers $\widetilde{\tilde{A}} \oplus \widetilde{\widetilde{B}}$

(ii) The difference of two interval-valued trapezoidal fuzzy numbers $\widetilde{\tilde{A}}-\widetilde{\tilde{B}}$ 


$$
\begin{aligned}
& \widetilde{\widetilde{A}}-\widetilde{\widetilde{B}}=\left[\left(a_{1}^{L}, a_{2}^{L}, a_{3}^{L}, a_{4}^{L} ; w_{\tilde{\tilde{A}} L}\right),\left(a_{1}^{U}, a_{2}^{U}, a_{3}^{U}, a_{4}^{U} ; w_{\widetilde{\tilde{A}}}\right)\right] \\
& -\left[\left(b_{1}^{L}, b_{2}^{L}, b_{3}^{L}, b_{4}^{L} ; w_{\tilde{B}}\right),\left(b_{1}^{U}, b_{2}^{U}, b_{3}^{U}, b_{4}^{U} ; w_{\tilde{B}}\right)\right] \\
& =\left[\begin{array}{c}
\left(a_{1}^{L}-b_{4}^{L}, a_{2}^{L}-b_{3}^{L}, a_{3}^{L}-b_{2}^{L}, a_{4}^{L}-b_{1}^{L} ; \min \left(w_{\tilde{A}}^{w_{\tilde{A}}}, w_{\tilde{B}}\right)\right), \\
\left(a_{1}^{U}-b_{4}^{U}, a_{2}^{U}-b_{3}^{U}, a_{3}^{U}-b_{2}^{U}, a_{4}^{U}-b_{1}^{U} ; \min (\underbrace{w_{\tilde{B}}}_{\tilde{\tilde{A}}}, w_{\tilde{B}})\right)
\end{array}\right]
\end{aligned}
$$

(iii) The product of two interval-valued trapezoidal fuzzy numbers $\widetilde{\tilde{A}} \otimes \widetilde{\widetilde{B}}$

$$
\begin{aligned}
& \widetilde{\tilde{A}} \otimes \widetilde{\tilde{B}}=\left[\left(a_{1}^{L}, a_{2}^{L}, a_{3}^{L}, a_{4}^{L} ; w_{\tilde{A}}\right),\left(a_{1}^{U}, a_{2}^{U}, a_{3}^{U}, a_{4}^{U} ; w_{\tilde{\tilde{A}}}\right)\right] \\
& \otimes\left[\left(b_{1}^{L}, b_{2}^{L}, b_{3}^{L}, b_{4}^{L} ; w_{\tilde{B}}\right),\left(b_{1}^{U}, b_{2}^{U}, b_{3}^{U}, b_{4}^{U} ; w_{\tilde{B}}\right)\right] \\
& =\left[\begin{array}{c}
\left(a_{1}^{L} \times b_{1}^{L}, a_{2}^{L} \times b_{2}^{L}, a_{3}^{L} \times b_{3}^{L}, a_{4}^{L} \times b_{4}^{L} ; \min \left(w_{\tilde{\tilde{A}} L}, w_{\tilde{B}}\right)\right), \\
\left(a_{1}^{U} \times b_{1}^{U}, a_{2}^{U} \times b_{2}^{U}, a_{3}^{U} \times b_{3}^{U}, a_{4}^{U} \times b_{4}^{U} ; \min \left(w_{\tilde{\tilde{A}}}, w_{\tilde{B}}\right)\right)
\end{array}\right]
\end{aligned}
$$

(iv) The product between an interval-valued trapezoidal fuzzy number and a constant $\lambda \widetilde{\tilde{A}}$

$$
\begin{aligned}
\lambda \widetilde{\widetilde{A}} & =\lambda \times\left[\left(a_{1}^{L}, a_{2}^{L}, a_{3}^{L}, a_{4}^{L} ; w_{\tilde{\tilde{A}}}\right),\left(a_{1}^{U}, a_{2}^{U}, a_{3}^{U}, a_{4}^{U} ; w_{\widetilde{A}}\right)\right] \\
& =\left[\left(\lambda a_{1}^{L}, \lambda a_{2}^{L}, \lambda a_{3}^{L}, \lambda a_{4}^{L} ; w_{\tilde{A}}^{L}\right),\left(\lambda a_{1}^{U}, \lambda a_{2}^{U}, \lambda a_{3}^{U}, \lambda a_{4}^{U} ; w_{\tilde{\tilde{A}}}\right)\right], \lambda>0
\end{aligned}
$$

(v) The division between two interval-valued trapezoidal fuzzy numbers $\widetilde{\tilde{A}} / \widetilde{\tilde{B}}$

$$
\begin{aligned}
& \frac{\widetilde{\tilde{A}}}{\widetilde{\widetilde{B}}}=\frac{\left[\left(a_{1}^{L}, a_{2}^{L}, a_{3}^{L}, a_{4}^{L} ; w_{\widetilde{\tilde{A}}}\right),\left(a_{1}^{U}, a_{2}^{U}, a_{3}^{U}, a_{4}^{U} ; w_{\widetilde{\tilde{A}}}\right)\right]}{\left[\left(b_{1}^{L}, b_{2}^{L}, b_{3}^{L}, b_{4}^{L} ; w_{\widetilde{\mathcal{B}}}\right),\left(b_{1}^{U}, b_{2}^{U}, b_{3}^{U}, b_{4}^{U} ; w_{\widetilde{\tilde{B}}}\right)\right]} \\
& =\left[\begin{array}{c}
\left(\min \left(U^{L}\right), \min \left(\frac{U^{L}}{x^{L}}\right), \max \left(\frac{U^{L}}{y^{L}}\right), \max \left(U^{L}\right) ; \min \left(w_{\widetilde{\tilde{A}}}, w_{\widetilde{B} \mathcal{L}}\right)\right), \\
\left(\min \left(U^{U}\right), \min \left(\frac{U^{U}}{x^{U}}\right), \max \left(\frac{U^{U}}{y^{U}}\right), \max \left(U^{U}\right) ; \min \left(w_{\widetilde{A} U}, w_{\widetilde{B} U}\right)\right)
\end{array}\right]
\end{aligned}
$$

Here,

$$
\begin{aligned}
U^{L} & =\left\{\frac{a_{1}{ }^{L}}{b_{1}{ }^{L}}, \frac{a_{2}{ }^{L}}{b_{2}{ }^{L}}, \frac{a_{3}{ }^{L}}{b_{3}{ }^{L}}, \frac{a_{4}{ }^{L}}{b_{4}{ }^{L}}\right\}, \\
U^{L} & =\left\{\frac{a_{1}{ }^{U}}{b_{1}{ }^{U}}, \frac{a_{2}{ }^{U}}{b_{2}{ }^{U}}, \frac{a_{3}{ }^{U}}{b_{3}{ }^{U}}, \frac{a_{4}{ }^{U}}{b_{4}{ }^{U}}\right\}, \\
x^{L}=\min \left(U^{L}\right), x^{U} & =\min \left(U^{U}\right), y^{L}=\max \left(U^{L}\right), y^{U} \max \left(U^{U}\right),
\end{aligned}
$$

and the operator " $/$ " denotes exclusion of a certain term from sets $U^{L}$ and $U^{U}$.

(vi) Rising to the power of a constant $\lambda$

$$
\begin{aligned}
\tilde{\tilde{A}}^{\lambda}= & {\left[\left(a_{1}^{L}, a_{2}^{L}, a_{3}^{L}, a_{4}^{L} ; w_{\tilde{\tilde{A}}}\right),\left(a_{1}^{U}, a_{2}^{U}, a_{3}^{U}, a_{4}^{U} ; w_{\widetilde{\tilde{A}}}\right)\right]^{\lambda} } \\
& \cdot\left[\left(\left(a_{1}^{L}\right)^{\lambda},\left(a_{2}^{L}\right)^{\lambda},\left(a_{3}^{L}\right)^{\lambda},\left(a_{4}^{L}\right)^{\lambda}, w_{\tilde{A} L}\right),\left(\left(a_{1}^{U}\right)^{\lambda},\left(a_{2}^{U}\right)^{\lambda},\left(a_{3}^{U}\right)^{\lambda},\left(a_{4}^{U}\right)^{\lambda}, w_{\tilde{\tilde{A}} U}\right)\right]
\end{aligned}
$$

By considering (Equation (3)), we can define the COG point for an interval-valued trapezoidal fuzzy number $\widetilde{\tilde{A}}=[$ $\left.\tilde{\tilde{A}}^{L}, \widetilde{\tilde{A}}^{U}\right]=\left[\left(a_{1}^{L}, a_{2}^{L}, a_{3}^{L}, a_{4}^{L} ; w_{\tilde{\tilde{A}}}^{L}\right),\left(a_{1}^{U}, a_{2}^{U}, a_{3}^{U}, a_{4}^{U} ; w_{\tilde{\tilde{A}}}\right)\right]$.

Firstly, Equation (3) is employed to obtain the coordinates of the COG points for the lower and upper values of $\widetilde{\tilde{A}}$, viz., ( $\left.x_{\tilde{A}}, y_{\tilde{A}}\right)$ and $\left(x_{\tilde{A} U}, y_{\tilde{A} U}\right)$ for $\tilde{\tilde{A}}^{L}$ and $\widetilde{\tilde{A}}^{U}$, respectively. Secondly, the COG of $\left(x_{\tilde{A}}, y_{\tilde{A}}\right)$ is found as follows:

$$
\left\{\begin{array}{l}
x_{\tilde{A}}=\frac{\left(\begin{array}{c}
x_{\tilde{A}}+x_{\tilde{A} U} \\
\tilde{A}
\end{array}\right)}{2}, \\
y_{\tilde{A}}=\frac{\left(\begin{array}{c}
y_{\tilde{A} L}+y_{\tilde{A} U} \\
\tilde{A}
\end{array}\right)}{2} .
\end{array}\right.
$$

Let there exist an interval-valued fuzzy number.

$$
\widetilde{\widetilde{B}}=\left[\widetilde{\widetilde{B}}^{L}, \widetilde{\widetilde{B}}^{U}\right]=\left[\left(b_{1}^{L}, b_{2}^{L}, b_{3}^{L}, b_{4}^{L} ; w_{\tilde{B}}\right),\left(b_{1}^{U}, b_{2}^{U}, b_{3}^{U}, b_{4}^{U} ; w_{\widetilde{B}}\right)\right] .
$$

One can define the COG point $\left(\underset{\tilde{B}}{x_{\tilde{B}}}, y_{\tilde{B}}\right)$ in the spirit of Equation (3). The distance $d_{\tilde{\tilde{A}}}$ and $d_{\tilde{B}}$ between the origin point and two generalized interval-valued trapezoidal fuzzy numbers $\widetilde{\tilde{A}}$ and $\widetilde{\widetilde{B}}$

$$
\widetilde{\widetilde{A}}=\left[\tilde{\widetilde{A}}^{L}, \widetilde{\tilde{A}}^{U}\right]=\left[\left(a_{1}^{L}, a_{2}^{L}, a_{3}^{L}, a_{4}^{L} ; w_{\tilde{A}}\right),\left(a_{1}^{U}, a_{2}^{U}, a_{3}^{U}, a_{4}^{U} ; w_{\tilde{A} U}\right)\right],
$$


and

$$
\widetilde{\widetilde{B}}=\left[\widetilde{\tilde{B}}^{L}, \widetilde{\widetilde{B}}^{U}\right]=\left[\left(b_{1}^{L}, b_{2}^{L}, b_{3}^{L}, b_{4}^{L} ; w_{\tilde{B}}\right),\left(b_{1}^{U}, b_{2}^{U}, b_{3}^{U}, b_{4}^{U} ; w_{\tilde{B}}^{U}\right)\right],
$$
tance:

respectively, are calculated by virtue of the Euclidean dis-

$$
\begin{aligned}
& d \underset{\tilde{A}}{\sim}=\sqrt{x_{\tilde{A}}^{2}+y_{\tilde{A}}^{2}}, \\
& d_{\tilde{B}}^{\sim}=\sqrt{x_{\tilde{B}}^{2}+y_{\tilde{B}}^{2}} .
\end{aligned}
$$

Accordingly, if $\underset{\tilde{A}}{d \sim} \underset{\tilde{B}}{\alpha}$, then $\tilde{\widetilde{A}}>\widetilde{\widetilde{B}}$.

The COG coordinates can also be employed when estimating the distance between two interval-valued trapezoidal fuzzy numbers say $\widetilde{\widetilde{A}}$ and $\widetilde{\widetilde{B}}[50,53]$.



Alternatively, one can employ the following technique (Liu and Jin, 2012):

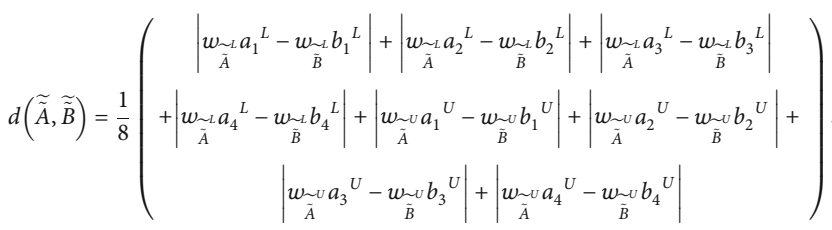

4.3. The Generalized Interval-Valued Trapezoidal Fuzzy Number Ordered Weighted Geometric Average Operator (OWGAO). [52] proposed an interesting and wellgrounded approach, named the ordered weighted average (OWA), which enabled to aggregate the variables in terms of their order in the set. Such an approach enables to avoid the subjectivity arising from group decision-making. [51] and later [24] introduced the generalized interval-valued trapezoidal fuzzy number ordered weighted geometric (GIFN-OWG) operator, which was able to tackle fuzzy ratings at $1^{\text {st }}$ level. But in the presented research work, the (GIFN-OWGA) is merged with average fuzzy rule and modified with renaming generalized interval-valued trapezoidal fuzzy number ordered weighted geometric average (GIFNOWG) operator.

fuzzy

Let $\widetilde{\tilde{A}}_{j}$ be a set of generalized interval-valued trapezoidal numbers,-

$\tilde{\tilde{A}}_{j k}=\left[\widetilde{\tilde{A}}_{j k}^{L}, \widetilde{\tilde{A}}_{j k}^{U}\right]=\left[\left(a_{1 j k}^{L}, a_{2 j k}^{L}, a_{3 j k}^{L}, a_{4 j k}^{L} ; \widetilde{\tilde{w}}_{A_{j k}^{L}}\right),\left(a_{1 j k}^{U}, a_{2 j k}^{U}, a_{3 j k}^{U}\right.\right.$,

$\left.\left.a_{4 j k}^{U} ; \widetilde{\tilde{w}}_{A_{j k}^{U}}\right)\right]$, with $j k=1,2,3, \cdots, n_{;} \Omega$ is the set of all general- ized interval-valued trapezoidal fuzzy numbers; $(\sigma(1), \sigma(2)$ $, \cdots, \sigma(n))$ is a permutation of $(1,2,3, \ldots, n)$, such that $\widetilde{\tilde{A}}_{\sigma(j k-1)}>\widetilde{\tilde{A}}_{\sigma(j k)}, \forall j k=2,3, \cdots, n$. Then, we have GITFNOWGA: $\Omega^{n} \longrightarrow \Omega$, which can be employed in the following way:

$$
\begin{aligned}
\operatorname{GITFNOWGA}_{w}\left(\widetilde{\tilde{A}}_{1}, \widetilde{\tilde{A}}_{2}, \cdots, \widetilde{\tilde{A}}_{n}\right)=\frac{\prod_{j k=1}^{n}\left(\widetilde{\tilde{A}}_{\sigma(j k)}\right)^{W_{j k}}}{\sum_{j k}^{n} \xi_{j k}} \\
=\prod_{j k=1}^{n}\left[\left(a_{1 \sigma(j k)}^{L}, a_{2 \sigma(j k)}^{L}, a_{3 \sigma(j k)}^{L}, a_{4 \sigma(j k)}^{L} ; w_{\tilde{A}_{\sigma(j k)}}\right),\right. \\
\left.\times\left(a_{1 \sigma(j k)}^{U}, a_{2 \sigma(j k)}^{U}, a_{3 \sigma(j k)}^{U}, a_{4 \sigma(j k)}^{U} ; w_{\tilde{A}_{\sigma(j k)}^{U}}\right)\right]^{W_{j k}} .
\end{aligned}
$$

Here, $w_{j k}$ is a weight of subdimensions to the $j k_{\text {th }}$ largest variable $(j k=1,2, \cdots, n)$. The vector of weights can be obtained by virtue of the following equation [62]:

$$
w_{j k+1}=\frac{C_{n-1}^{i}}{2^{n-1}}, \forall i=0,1,2,3 \cdots, n-1 .
$$

\section{The Crisp MULTI-MOORA Algorithm}

The Multiobjective Optimization by Ratio Analysis (MOORA) algorithm was introduced by [43], Sahu et al. [24], Bu et al. [40], and $\mathrm{He}$ et al. [41] and extended the algorithm to make it more robust as MULTI-MOORA (MOORA plus the full multiplicative form).

The MOORA algorithm begins with matrix $X$ where its elements $x_{i j}$ denote $i_{\text {th }}$ alternative of $j_{\text {th }}$ objective $(i=1,2$, $\cdots, m ; j=1,2, \cdots, n)$. The MOORA algorithm consists of two parts: the ratio system and the reference point approach. The MULTI-MOORA algorithm includes internal normalization and treats originally all the objectives equally important. In principle, all stakeholders interested in the issue only could give more importance to an objective. Therefore, they could either multiply the dimensionless number representing the response on an objective with a significance coefficient or they could decide beforehand to split an objective into different subobjectives.

5.1. The Ratio System of MOORA. Ratio system defines data normalization by comparing alternative of an objective to all values of the objective:

$$
x_{i j}^{*}=\frac{x_{i j}}{\sqrt{\sum_{i=1}^{m} x_{i j}^{2}}} .
$$

Here, $x_{i j}^{*}$ denotes $i_{\text {th }}$ alternative of $j_{\text {th }}$ objective. Usually these numbers belong to the interval $[0,1]$. These indicators are added (if desirable value of indicator is maximum) or subtracted (if desirable value is minimum); thus, the 


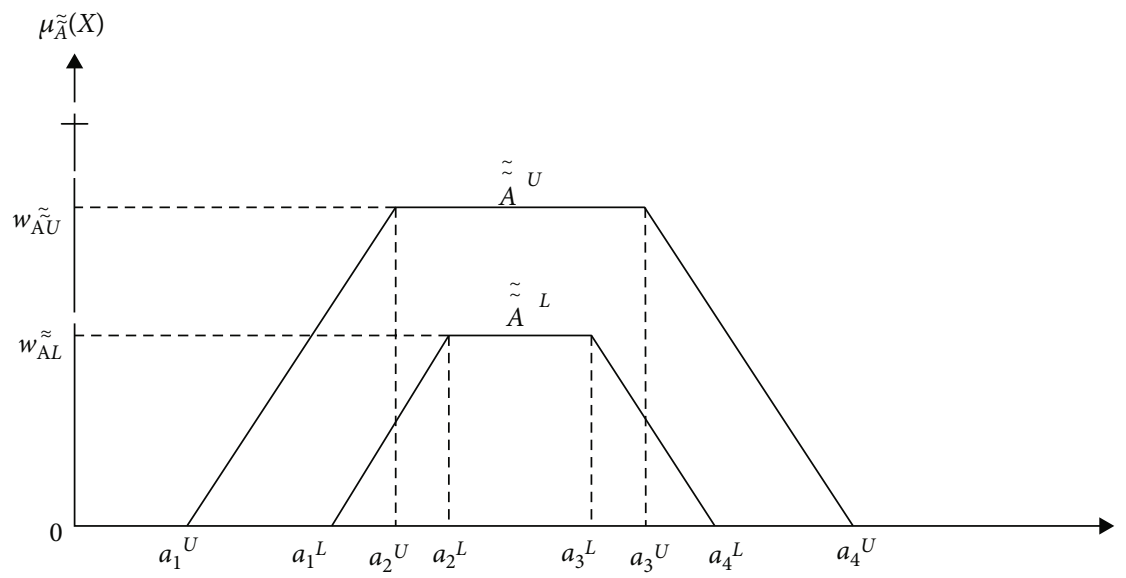

FIgURE 3: Interval-valued trapezoidal fuzzy numbers.

summarizing index of each alternative is derived in this way:

$$
y_{i}^{*}=\sum_{j=1}^{g} x_{i j}^{*}-\sum_{j=g+1}^{n} x_{i j}^{*} .
$$

Here, $g=1, \cdots, n$ denotes number of objectives to be maximized. Then, every ratio is given the rank: the higher the index, the higher the rank.

In some cases, it is often observed that some attributes are more important than the others. In order to give more importance to an attribute, it could be multiplied with its corresponding weight (significance coefficient) [38, 42, 62]. When these attribute weights are taken into consideration, Equation (28) becomes as follows:

$$
y_{i}^{*}=\sum_{j=1}^{g} w_{j} x_{i j}^{*}-\sum_{j=g+1}^{n} w_{j} x_{i j}^{*}, j=1,2, \cdots, n .
$$

Here, $w_{j}$ is the weight of $j_{\text {th }}$ subdimensions.

5.2. The Reference Point of MOORA. Reference point approach is based on the ratio system. The Maximal Objective Reference Point (vector) is found according to ratios found by employing Eq. The $j_{\text {th }}$ coordinate of the reference point can be described as $\left(r_{j}=\max x_{i j}^{*}\right)$ in the case of maximization. Every coordinate of this vector represents maximum or minimum of certain objective (indicator). Then, every element of normalized response matrix is recalculated, and the final rank is given according to deviation from the reference point and the Min-Max Metric of Tchebycheff:

$$
\min _{i}\left(\max _{j} \underset{j}{r_{j}-x_{i j}^{*} \mid}\right) \text {. }
$$

5.3. The Full Multiplicative Form and MULTI-MOORA. [43] proposed MOORA to be updated by the full multiplicative form algorithm embodying maximization as well as minimization of purely multiplicative utility function. Overall utility of the $i_{\text {th }}$ alternative can be expressed as dimensionless number:

$$
U_{i}^{\prime}=\frac{A_{i}}{B_{i}}
$$

Here, $A_{i}=\prod_{j=1}^{g} x_{i j} ; i=1,2, \cdots, m$ denotes the product of objectives of the $i_{\text {th }}$ alternative to be maximized with $g=1$, $2, \cdots, n$ being the number of objectives to be maximized and where $B_{i}=\prod_{j=g+1}^{n} x_{i j} ; i=1,2, \cdots, m$ denotes the product of objectives of the $i_{\text {th }}$ alternative to be minimized with $n-g$ being the number of objectives (indicators) to be minimized. Thus, MULTI-MOORA summarizes MOORA (i.e., ratio system and reference point) and the full multiplicative form.

5.4. MULTI-MOORA Algorithm Based upon Interval-Valued Trapezoidal Fuzzy Numbers. Let $E=1,2, \cdots, e$ denote the $e_{\text {th }}$ expert involved in a decision-making process. Suppose that the experts provide ratings for each $i_{\text {th }}$ alternative against each $j_{\text {th }}$ criterion with $i=1,2, \cdots, m$ and $j=1,2, \cdots, n$. The set of criteria can be split into two subsets, namely, those of cost criteria, $C$, and benefit criteria, $B$. Cost criteria are to be minimized, whereas benefit criteria are to be maximized. Each criterion can be attributed with respective weight $W_{j}$, such that $W_{j} \geq 0$, and $\sum_{j} W_{j}=1$.

Step 1. Each of decision-makers constructs his own decision matrix: $\left(\widetilde{\tilde{A}}^{k}\right)_{m \times n}$ with elements $\widetilde{\tilde{a}}_{i j}^{k}=\left[\left(a_{i j k 1}^{L}, a_{i j k 2}^{L}, a_{i j k 3}^{L}, a_{i j k 4}^{L}\right.\right.$; $\left.\left.w_{i j k}^{L}\right),\left(a_{i j k 1}^{U}, a_{i j k 2}^{U}, a_{i j k 3}^{U}, a_{i j k 4}^{U} ; w_{i j k}^{U}\right)\right]$ being responses of alternatives on criteria. 
Table 1: Dynamic multidimension Coal Refinery Process Absorbability Index (CRPAI) structure.

\begin{tabular}{|c|c|c|}
\hline Coal refinery process, $(\mathrm{C})$ & Dimensions; $\xi_{j}$ & Subdimensions; $\xi_{j k}$ \\
\hline \multirow{8}{*}{$\begin{array}{l}\text { Coal Refinery Process Absorbability } \\
\text { Index evaluation }\end{array}$} & Absorbability index on crushing and breaking process, $\xi_{1}$ & $\begin{array}{l}\text { Effective primary breaking with low gas } \\
\text { emission, } \xi_{11} \\
\text { Effective final breaking with low gas } \\
\text { emission, } \xi_{12}\end{array}$ \\
\hline & \multirow[b]{2}{*}{$\begin{array}{c}\text { Absorbability index on storage and stockpiling process } \\
\text { and density separation, } \xi_{3}\end{array}$} & $\begin{array}{l}\text { Effective machining for sizing with low } \\
\text { gas emission, } \xi_{21}\end{array}$ \\
\hline & & $\begin{array}{l}\text { Toxic gas emission on the stockpiling of } \\
\text { coal, } \xi_{31} \\
\text { Emission of toxic gases on density } \\
\text { separation, } \xi_{32}\end{array}$ \\
\hline & \multirow{3}{*}{$\begin{array}{l}\text { Absorbability index on density separation and washing } \\
\text { process, } \xi_{4}\end{array}$} & $\begin{array}{l}\text { Emission of toxic gases on quenching } \\
\text { process, } \xi_{41}\end{array}$ \\
\hline & & $\begin{array}{l}\text { Water prequenching treatment level, } \\
\qquad \xi_{42}\end{array}$ \\
\hline & & $\begin{array}{l}\text { Water final-quenching treatment level, } \\
\qquad \xi_{43}\end{array}$ \\
\hline & \multirow{2}{*}{ Absorbability index on drying process, $\xi_{5}$} & $\begin{array}{l}\text { Moisture drying pretreatment level, } \xi_{42} \text {, } \\
\xi_{51}\end{array}$ \\
\hline & & $\begin{array}{l}\text { Moisture drying final treatment level, } \\
\xi_{51}\end{array}$ \\
\hline
\end{tabular}

TABLE 2: Nine-member linguistic terms (rating scale) and their corresponding interval-valued fuzzy numbers.

\begin{tabular}{lc}
\hline Linguistic terms & Interval-valued trapezoidal fuzzy numbers \\
\hline Absolutely poor, AP & {$[(0.0,0.0,0.0,0.0 ; 0.5),(0.0,0.0,0.0,0.0 ; 1.0)]$} \\
Very poor, VP & {$[(0.0075,0.0075,0.015,0.0525 ; 0.5),(0.0,0.0,0.02,0.07 ; 1.0)]$} \\
Poor, P & {$[(0.0875,0.12,0.16,0.1825 ; 0.5),(0.04,0.10,0.18,0.23 ; 1.0)]$} \\
Fairly poor, FP & {$[(0.2325,0.255,0.325,0.3575 ; 0.5),(0.17,0.22,0.36,0.42 ; 1.0)]$} \\
Medium, M & {$[(0.4025,0.4525,0.5375,0.5676 ; 0.5),(0.32,0.41,0.58,0.65 ; 1.0)]$} \\
Fairly satisfactory, FS & {$[(0.65,0.6725,0.7575,0.79 ; 0.5),(0.58,0.63,0.80,0.86 ; 1.0)]$} \\
Satisfactory, S & {$[(0.7825,0.815,0.885,0.9075 ; 0.5),(0.72,0.78,0.92,0.97 ; 1.0)]$} \\
Very impressive, VI & {$[(0.9475,0.985,0.9925,0.9925 ; 0.5),(0.93,0.98,1.0,1.0 ; 1.0)]$} \\
Absolutely impressive, AI & {$[(1.0,1.0,1.0,1.0 ; 0.5),(1.0,1.0,1.0,1.0 ; 1.0)]$} \\
\hline
\end{tabular}

Step 2. Individual decision matrices are aggregated by employing the GITFNOWGA operator.

$$
\operatorname{GITFNOWGA}_{w}\left(\widetilde{\tilde{a}}_{i j}^{1}, \widetilde{\tilde{a}}_{i j}^{2}, \cdots, \widetilde{\tilde{a}}_{i j}^{K}\right)=\prod_{k=1}^{K}\left(a_{i j}^{\sigma(k)}\right)^{w_{k}}, \forall i, j
$$

Here, $w_{k}$ is the weight of the $k_{\text {th }}$ largest response obtained by Equation (26) and

$$
\tilde{\tilde{a}}_{i j}=\left[\left(a_{i j 1}^{L}, a_{i j 2}^{L}, a_{i j 3}^{L}, a_{i j 4}^{L} ; w_{i j}^{L}\right),\left(a_{i j 1}^{U}, a_{i j 2}^{U}, a_{i j 3}^{U}, a_{i j 4}^{U} ; w_{i j}^{U}\right)\right] .
$$

It must be noted that Equation (21) is employed to com- pare the values of the generalized interval-valued trapezoidal fuzzy numbers.

Step 3. In case some of criteria involve numeric data, the normalization has to be carried out.

$$
\begin{aligned}
\widetilde{\tilde{x}}_{i j} & =\left[\left(\frac{a_{i j 1}^{L}}{d_{j}}, \frac{a_{i j 2}^{L}}{d_{j}}, \frac{a_{i j 3}^{L}}{d_{j}}, \frac{a_{i j 4}^{L}}{d_{j}} ; w_{i j}^{L}\right),\left(\frac{a_{i j 1}^{U}}{d_{j}}, \frac{a_{i j 2}^{U}}{d_{j}}, \frac{a_{i j 3}^{U}}{d_{j}}, \frac{a_{i j 4}^{U}}{d_{j}} ; w_{i j}^{U}\right)\right] \\
& =\left[\left(x_{i j 1}^{L}, x_{i j 2}^{L}, x_{i j 3}^{L}, x_{i j 4}^{L} ; w_{i j}^{L}\right),\left(x_{i j 1}^{U}, x_{i j 2}^{U}, x_{i j 3}^{U}, x_{i j 4}^{U} ; w_{i j}^{U}\right)\right], j=1,2, \cdots, n ; i=1,2, \cdots, n .
\end{aligned}
$$

Here, $d_{j}=\sqrt{\sum_{i=1}^{m} \sum_{p=1}^{4}\left(a_{i j p}^{L}\right)^{2}+\sum_{i=1}^{m} \sum_{p=1}^{4}\left(a_{i j p}^{U}\right)^{2}}, p=\{1,2$ $, 3,4\}$ for $\forall j=1,2, \cdots, n$.

Step 4. The ratio system: 
TABLE 3: Appropriateness ratings (in linguistic scale) against CRPAI subdimensions assigned by EP.

\begin{tabular}{|c|c|c|c|c|c|c|}
\hline$\theta_{i}$ & $\xi_{i j}$ & $E_{1}$ & $E_{2}$ & $E_{3}$ & $E_{4}$ & $E_{5}$ \\
\hline \multirow{2}{*}{$\theta_{1}$} & $\xi_{11}$ & $\mathrm{~S}$ & $\mathrm{VI}$ & $\mathrm{M}$ & FS & $\mathrm{AI}$ \\
\hline & $\xi_{12}$ & VI & S & FS & $\mathrm{M}$ & AI \\
\hline \multirow{2}{*}{$\theta_{2}$} & $\xi_{11}$ & FS & S & $S$ & S & $\mathrm{S}$ \\
\hline & $\xi_{12}$ & S & S & $\mathrm{FS}$ & $\mathrm{S}$ & $\mathrm{S}$ \\
\hline \multirow{2}{*}{$\theta_{3}$} & $\xi_{11}$ & S & S & $S$ & VI & VI \\
\hline & $\xi_{12}$ & S & S & $\mathrm{S}$ & VI & VI \\
\hline \multirow{2}{*}{$\theta_{4}$} & $\xi_{11}$ & $\mathrm{M}$ & $\mathrm{M}$ & $\mathrm{M}$ & $\mathrm{M}$ & $\mathrm{M}$ \\
\hline & $\xi_{12}$ & $\mathrm{M}$ & $\mathrm{M}$ & $\mathrm{M}$ & $\mathrm{M}$ & $\mathrm{M}$ \\
\hline$\theta_{1}$ & $\xi_{21}$ & FS & S & FS & S & FS \\
\hline$\theta_{2}$ & $\xi_{21}$ & FP & $\mathrm{M}$ & $\mathrm{M}$ & $\mathrm{M}$ & $\mathrm{M}$ \\
\hline$\theta_{3}$ & $\xi_{21}$ & FS & S & FS & FS & FS \\
\hline$\theta_{4}$ & $\xi_{21}$ & $S$ & $\mathrm{~S}$ & $\mathrm{VI}$ & VI & $\mathrm{S}$ \\
\hline \multirow{2}{*}{$\theta_{1}$} & $\xi_{31}$ & $\mathrm{AI}$ & $\mathrm{AI}$ & $\mathrm{AI}$ & VI & VI \\
\hline & $\xi_{32}$ & AI & $\mathrm{AI}$ & $\mathrm{AI}$ & VI & VI \\
\hline \multirow{2}{*}{$\theta_{2}$} & $\xi_{31}$ & $S$ & VI & VI & VI & VI \\
\hline & $\xi_{32}$ & S & VI & VI & VI & VI \\
\hline \multirow{2}{*}{$\theta_{3}$} & $\xi_{31}$ & FS & S & S & S & $\mathrm{S}$ \\
\hline & $\xi_{32}$ & FS & S & $\mathrm{S}$ & S & S \\
\hline \multirow{2}{*}{$\theta_{4}$} & $\xi_{31}$ & S & S & $\mathrm{S}$ & VI & VI \\
\hline & $\xi_{32}$ & S & S & $\mathrm{S}$ & VI & VI \\
\hline \multirow{3}{*}{$\theta_{1}$} & $\xi_{41}$ & $\mathrm{M}$ & $\mathrm{M}$ & S & VI & $\mathrm{M}$ \\
\hline & $\xi_{42}$ & $\mathrm{M}$ & $\mathrm{M}$ & $S$ & VI & $\mathrm{M}$ \\
\hline & $\xi_{41}$ & $\mathrm{M}$ & $\mathrm{M}$ & $S$ & VI & $\mathrm{M}$ \\
\hline \multirow{3}{*}{$\theta_{2}$} & $\xi_{41}$ & FS & S & $\mathrm{M}$ & $\mathrm{M}$ & FS \\
\hline & $\xi_{42}$ & FS & S & $\mathrm{M}$ & $\mathrm{M}$ & FS \\
\hline & $\xi_{43}$ & FS & S & $\mathrm{M}$ & $\mathrm{M}$ & FS \\
\hline \multirow{3}{*}{$\theta_{3}$} & $\xi_{41}$ & FP & $\mathrm{M}$ & S & S & $\mathrm{M}$ \\
\hline & $\xi_{42}$ & FP & $\mathrm{M}$ & $\mathrm{S}$ & S & $\mathrm{M}$ \\
\hline & $\xi_{43}$ & $\mathrm{FP}$ & $\mathrm{M}$ & $\mathrm{S}$ & $\mathrm{S}$ & $\mathrm{M}$ \\
\hline \multirow{3}{*}{$\theta_{4}$} & $\xi_{41}$ & S & FS & $\mathrm{M}$ & $\mathrm{M}$ & FS \\
\hline & $\xi_{42}$ & FS & S & $\mathrm{M}$ & $\mathrm{M}$ & FS \\
\hline & $\xi_{43}$ & FS & S & $\mathrm{M}$ & $\mathrm{M}$ & FS \\
\hline \multirow{2}{*}{$\theta_{1}$} & $\xi_{51}$ & $\mathrm{AI}$ & $\mathrm{AI}$ & AI & VI & VI \\
\hline & $\xi_{52}$ & VI & $\mathrm{AI}$ & AI & AI & $\mathrm{VI}$ \\
\hline \multirow{2}{*}{$\theta_{2}$} & $\xi_{51}$ & S & VI & $\mathrm{S}$ & VI & VI \\
\hline & $\xi_{52}$ & S & VI & $\mathrm{S}$ & VI & VI \\
\hline \multirow{2}{*}{$\theta_{3}$} & $\xi_{51}$ & FS & S & S & FS & $\mathrm{S}$ \\
\hline & $\xi_{52}$ & FS & S & $S$ & FS & $S$ \\
\hline
\end{tabular}

TABLE 3: Continued.

\begin{tabular}{ccccccc}
\hline$\theta_{i}$ & $\xi_{i j}$ & $E_{1}$ & $E_{2}$ & $E_{3}$ & $E_{4}$ & $E_{5}$ \\
\hline \multirow{2}{*}{$\theta_{4}$} & $\xi_{51}$ & $\mathrm{~S}$ & $\mathrm{~S}$ & $\mathrm{~S}$ & $\mathrm{VI}$ & $\mathrm{VI}$ \\
& $\xi_{52}$ & $\mathrm{VI}$ & $\mathrm{S}$ & $\mathrm{S}$ & $\mathrm{S}$ & $\mathrm{VI}$ \\
\hline
\end{tabular}

The normalized values are added up for the benefit criteria and subtracted for the cost criteria:

$$
\begin{aligned}
\mathrm{RS}_{i}= & \sum_{j \in B} \widetilde{\tilde{x}}_{i j}-\sum_{j \in C} \widetilde{\tilde{x}}_{i j}=\left[\left(\mathrm{RS}_{i 1}^{L}, \mathrm{RS}_{i 2}^{L}, \mathrm{RS}_{i 3}^{L}, \mathrm{RS}_{i 4}^{L} ; w_{\mathrm{RS}_{i}}^{L}\right)\right. \\
& \left.\cdot\left(\mathrm{RS}_{i 1}^{U}, \mathrm{RS}_{i 2}^{U}, \mathrm{RS}_{i 3}^{U}, \mathrm{RS}_{i 4}^{U} ; w_{\mathrm{RS}_{i}}^{U}\right)\right]
\end{aligned}
$$

Here, $\mathrm{RS}_{i}$ denotes the overall utility of the $i_{\text {th }}$ alternative in terms of the ratio system. The alternatives are then ranked by measuring their distances from the origin point in the spirit of Equation (21). Specially, alternatives with higher distances receive higher ranks.

Step 5. The reference point approach:

For the sake of convenience, one can employ the Maximal Utopian Reference Point (MURP), rather than the Maximal Objective Reference Point. In the case of the generalized interval-valued trapezoidal fuzzy numbers, MURP is defined as follows:

$$
\widetilde{\tilde{r}}_{j}= \begin{cases}(1,1,1,1 ; 1), & \forall j \in B, \\ (0,0,0,0 ; 1), & \forall j \in C .\end{cases}
$$

Therefore, Equations (23) and (24) can be utilized to identify the maximal deviation from the MURP for each alternative:

$$
\max _{j} d\left(\widetilde{\tilde{r}}_{j}, \widetilde{\tilde{x}}_{i j}\right)
$$

Then, the alternatives are ranked by minimizing the maximal deviances found in Equation (36).

Step 6. The full multiplicative form:

The fuzzy utility of the $i_{\text {th }}$ alternative is obtained by employing Equations (12) and (14).

$$
\widetilde{\widetilde{U}}_{i}=\frac{\widetilde{\tilde{A}}_{i}}{\widetilde{\tilde{B}}_{i}}
$$

Here, $\widetilde{\tilde{A}}_{i}=\prod_{j \in B} \widetilde{\widetilde{x}}_{i j}, i=1,2, \cdots, m$ denotes the product of objectives of the $i_{\text {th }}$ alternative to be maximized with $B$ being the set of objectives to be maximized and where $\widetilde{\widetilde{B}}_{i}=\prod_{j \in C} \widetilde{\tilde{x}}_{i j}$ denotes the product of objectives of the $i_{\text {th }}$ alternative to be minimized with $C$ being the set of objectives (indicators) need to be minimized. The alternatives are ranked in descending order of $\widetilde{\tilde{U}}_{i}$ by employing Equation (21). 
TABLE 4: Computed aggregated fuzzy ratings against CRPAI subdimensions $\left(\xi_{j}\right)$.

\begin{tabular}{|c|c|c|}
\hline$\xi_{j}$ & $\theta_{i}$ & Aggregated fuzzy ratings against CRPAI subdimensions; $\xi_{j}$ \\
\hline \multirow{4}{*}{$\xi_{1}$} & $\theta_{1}$ & {$[(0.764,0.796,0.856,0.877,0.500),(0.706,0.765,0.887,0.928,1.000)]$} \\
\hline & $\theta_{2}$ & {$[(0.774,0.804,0.869,0.890,0.500),(0.716,0.772,0.901,0.947,1.000)]$} \\
\hline & $\theta_{3}$ & {$[(0.830,0.864,0.917,0.933,0.500),(0.779,0.837,0.944,0.979,1.000)]$} \\
\hline & $\theta_{4}$ & {$[(0.625,0.661,0.742,0.770,0.500),(0.550,0.621,0.781,0.840,1.000)]$} \\
\hline \multirow{4}{*}{$\xi_{2}$} & $\theta_{1}$ & {$[(0.688,0.697,0.715,0.721,0.500),(0.671,0.688,0.724,0.736,1.000)]$} \\
\hline & $\theta_{2}$ & {$[(0.389,0.437,0.522,0.552,0.500),(0.308,0.395,0.564,0.633,1.000)]$} \\
\hline & $\theta_{3}$ & {$[(0.657,0.659,0.662,0.663,0.500),(0.654,0.657,0.664,0.666,1.000)]$} \\
\hline & $\theta_{4}$ & {$[(0.830,0.864,0.917,0.933,0.500),(0.779,0.837,0.944,0.979,1.000)]$} \\
\hline \multirow{4}{*}{$\xi_{3}$} & $\theta_{1}$ & {$[(0.983,0.995,0.998,0.998,0.500),(0.978,0.994,1.000,1.000,1.000)]$} \\
\hline & $\theta_{2}$ & {$[(0.937,0.974,0.986,0.987,0.500),(0.916,0.967,0.995,0.998,1.000)]$} \\
\hline & $\theta_{3}$ & {$[(0.718,0.743,0.795,0.812,0.500),(0.668,0.717,0.820,0.857,1.000)]$} \\
\hline & $\theta_{4}$ & {$[(0.830,0.864,0.917,0.933,0.500),(0.779,0.837,0.944,0.979,1.000)]$} \\
\hline \multirow{4}{*}{$\xi_{4}$} & $\theta_{1}$ & {$[(0.500,0.549,0.632,0.660,0.500),(0.418,0.507,0.673,0.737,1.000)]$} \\
\hline & $\theta_{2}$ & {$[(0.567,0.589,0.624,0.636,0.500),(0.525,0.570,0.641,0.666,1.000)]$} \\
\hline & $\theta_{3}$ & {$[(0.479,0.525,0.609,0.639,0.500),(0.396,0.482,0.650,0.717,1.000)]$} \\
\hline & $\theta_{4}$ & {$[(0.830,0.864,0.917,0.933,0.500),(0.779,0.837,0.944,0.979,1.000)]$} \\
\hline \multirow{4}{*}{$\xi_{5}$} & $\theta_{1}$ & {$[(0.983,0.995,0.998,0.998,0.500),(0.978,0.994,1.000,1.000,1.000)]$} \\
\hline & $\theta_{2}$ & {$[(0.937,0.974,0.986,0.987,0.500),(0.916,0.967,0.995,0.998,1.000)]$} \\
\hline & $\theta_{3}$ & {$[(0.718,0.743,0.795,0.812,0.500),(0.668,0.717,0.820,0.857,1.000)]$} \\
\hline & $\theta_{4}$ & {$[(0.830,0.864,0.917,0.933,0.500),(0.779,0.837,0.944,0.979,1.000)]$} \\
\hline
\end{tabular}

TABLE 5: Preference orders computed by ratio system analysis algorithm.

\begin{tabular}{clcc}
\hline$\theta_{i}$ & \multicolumn{1}{c}{$\mathrm{RS}_{i}$} & $d_{A^{\tau}}$ & Preference orders \\
\hline$\theta_{1}$ & {$[(3.920,4.033,4.199,4.253,5.00),(3.751,3.947,4.283,4.401,1.000)]$} & 8.582 & 2 \\
$\theta_{2}$ & {$[(3.603,3.778,3.986,4.052,5.00),(3.380,3.669,4.095,4.242,1.000)]$} & 8.406 & 4 \\
$\theta_{3}$ & {$[(3.402,3.535,3.778,3.858,5.00),(3.167,3.411,3.899,4.075,1.000)]$} & 8.456 & 3 \\
$\theta_{4}$ & {$[(3.946,4.119,4.410,4.502,5.00),(3.668,3.970,4.558,4.757,1.000)]$} & 8.730 & 1 \\
\hline
\end{tabular}

Step 7. The dominance theory ([45], [40], [41]) is employed to aggregate the ranks, provided by respective parts of MULTI-MOORA.

\section{Empirical Research}

In the presented research forum, the authors developed a standard dynamic multidimension Coal Refinery Process Absorbability Index (CRPAI) structure by conducting the real CRP audit and the prior state of arts and proposed the same to CRP companies for finding the best CRP technique/practice to control further air pollution-global warming under discussed twice factors, shown in Table 1.

To simulate the CRPAI, the authors used modified OWGA operator with application of the MULTI-MOORA algorithm embedded with dominance theory. To validate the CRPAI, the authors solved a problem of a CRP company, existing north part of India. The CRP company wanted to
TABLE 6: Preference orders computed by reference point algorithm.

\begin{tabular}{ccc}
\hline$\theta_{i}$ & $\operatorname{Max} j\{d(\beta i j, \beta j)$ & Preference orders \\
\hline$\theta_{1}$ & 0.562 & 3 \\
$\theta_{2}$ & 0.644 & 4 \\
$\theta_{3}$ & 0.549 & 2 \\
$\theta_{4}$ & 0.476 & 1 \\
\hline
\end{tabular}

evaluate and finalize a reliable CRP alternative amongst available $\left(\theta_{1}, \theta_{2}, \theta_{3}\right.$, and $\left.\theta_{4}\right)$ for controlling further air pollution, occurs due to discussed twice factors. For data modeling of the subdimensions of CRPAI, a board of five highly experience decision-makers $E_{1}, E_{2} E_{3} E_{4}$, and $E_{5}$ are evaluated from the inventory, production, and environmental departments of CRP (case study) company. Next, the expert's panel is invited to sight, audit, and assess the 
TABLe 7: Preference orders computed by full multification form (MULTI-MOORA) algorithm.

\begin{tabular}{cccc}
\hline$\theta_{i}$ & \multicolumn{1}{c}{$y_{i}$} & $d_{A^{z}}$ & Preference orders \\
\hline$\theta_{1}$ & {$[(0.255,0.302,0.385,0.415,0.500),(0.189,0.263,0.432,0.503,1.000)]$} & 1.930 & 2 \\
$\theta_{2}$ & {$[(0.150,0.196,0.275,0.304,0.500),(0.097,0.162,0.322,0.398,1.000)]$} & 1.908 & 4 \\
$\theta_{3}$ & {$[(0.135,0.165,0.234,0.260,0.500),(0.090,0.136,0.274,0.343,1.000)]$} & 1.919 & 3 \\
$\theta_{4}$ & {$[(0.297,0.369,0.524,0.583,0.500),(0.203,0.305,0.621,0.772,1.000)]$} & 1.963 & 1 \\
\hline
\end{tabular}

capability of alternative CRPs such as $\theta_{1}, \theta_{2}, \theta_{3}$, and $\theta_{4}$ with high concern about the discussed twice factors. After that, decision-makers are assisted with linguistic variable scale, expressed 1-9 IVFN scale as shown in Table 2, so that decision-makers can assign appropriateness ratings against individual subdimensions.

The procedural steps are summarized below:

Step 1. Gathering information from the expert group in relation to performance ratings of different evaluation dimensions and subdimensions using linguistic terms:

In order to acquire appropriateness ratings against subdimensions, evaluated board expert's panel, e.g., $E_{1}, E_{2}, E_{3}$, $E_{4}$, and $E_{5}$, instructed to assign appropriateness ratings (evaluation score) against subdimensions (associated in proposed structure), which is further transformed into IV-fuzzy number. The appropriateness ratings (in linguistic terms) against evaluated subdimensions assigned by the expert panel corresponding to preferred options, e.g., $\theta_{1}, \theta_{2}, \theta_{3}$, and $\theta_{4}$, have depicted in Table 3.

Step 2. Approximation of the linguistic evaluation information by IV trapezoidal fuzzy numbers:

The assigned appropriateness ratings acquired in the form of IVFNs against subdimensions, shown in Table 3, are shifted into $1^{\text {st }}$ level (core dimensions) by using modified GITFN-OWGAO (generalized interval-valued trapezoidal fuzzy number ordered weighted geometric average operator); In Equations (25) and (26), consequently, the problem appeared as structure modeling, revealed in Table 4.

$$
\begin{aligned}
& \xi_{1} \quad \xi_{1} \quad \xi_{3} \quad \xi_{j}
\end{aligned}
$$

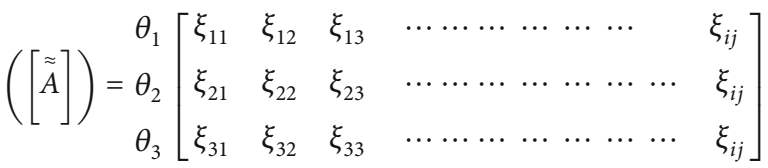

Step 3. Normalization:

After forming structure matrix, the IV fuzzy set value against core dimensions is found out in between $[0,1]$ and core dimensions were beneficial in nature, Hence, structural matrix was not normalized with exploration of Equation (34).

Step 4. The ratio system:

In the ratio system (RS), the values of IVFNs against core dimensions corresponding to CRP alternative or techniques
TABLE 8: CRPAI of CR process.

\begin{tabular}{ccccc}
\hline$\theta_{i}$ & $\begin{array}{c}\text { Ratio } \\
\text { system }\end{array}$ & $\begin{array}{c}\text { Reference } \\
\text { point }\end{array}$ & $\begin{array}{c}\text { Full multification } \\
\text { form }\end{array}$ & $\begin{array}{c}\text { Dominance } \\
\text { theory }\end{array}$ \\
\hline$\theta_{1}$ & 2 & 3 & 2 & 2 \\
$\theta_{2}$ & 4 & 4 & 4 & 4 \\
$\theta_{3}$ & 3 & 2 & 3 & 3 \\
$\theta_{4}$ & 1 & 1 & 1 & 1 \\
\hline
\end{tabular}

are added up for the benefit criteria and subtracted for the cost criteria with exploration of Equation (35); the results are shown in Table 5. The higher scores are prioritized corresponds to higher preference orders.

Step 5. Reference point approach:

We define the reference point (ascertained the core dimensions are benefit in nature)

$$
\widetilde{\widetilde{r}}_{i}=(1,1,1,1 ; 1) .
$$

Thus, the preference order of preferred CRPs is decided in accordance to their distances from fuzzy reference points and is computed by using Equations (36) and (37). Smaller distances of subdimensions from fuzzy reference points are preferred first, shown in Table 6.

Step 6. The full multiplicative form:

Eventually, Equation (38) is utilized to obtain preference orders of CRP options in accordance to full multiplicative form of MOORA, and the results are shown in Table 7.

Step 7. Final ranking orders by exploring of dominance theory:

By passing through respective computational algorithms of MULTI-MOORA: ratio system analysis, reference point, and full multiplicative form, finally the dominance theory ([45]; [40], [41]) is implemented to summarize the preference orders provided by three respective parts of MULTIMOORA computational algorithms $\mathrm{Bu}$ et al. [40] and $\mathrm{He}$ et al. [41]. Hence, the final preference orders of preferred CRP options are presented in Table 8.

As results, the fourth CRP practice/technique/process ( $\left.\theta_{4}\right)$ is found more viable amongst preferred CRP practices/techniques/processes as per the subjective report of expert panel, whereas the first CRP practice/technique/process $\left(\theta_{1}\right.$ ) and subsequently $\left(\theta_{3}\right)$ are found as a second and third best 
CRP practice/technique/process. At the other end of spectrum, second coal partner $\left(\theta_{2}\right)$ has regrettably found as a worst CRP practice/technique/process, and final preference orders are depicted as $\theta_{4}>\theta_{1}>\theta_{3}>\theta_{2}$.

It was visibly found that $\left(\theta_{4}\right)$ CRP technique/process would be the best to diminish the air pollution, growing universal warming and preserving the ecological balance caused by CRPs said by ([7, 19, 20, 22, 28, 48, 63]; Langergraber).

\section{Novelties of Conducted Research}

(i) The authors served a research clue in the front of CRP researchers to overcome global warming issue by addressing the discussed twice factors

(ii) The authors proposed and developed CRPAI structure (consisted of coal refinery core dimension and subdimensions corresponding to CRP alternative) to diminish the air pollution

(iii) This research dealt with comprehensive qualitative modeling of dimension and subdimensions corresponding to CRP alternative using expert information

(iv) These authors adapted and applied the three qualitative data optimization algorithms concurrently to robustly opt the viable CRP $[40,41]$

(v) The authors modified the IVFN-OWGA (intervalvalued fuzzy number ordered weighted geometric average) operator by introducing the average fuzzy rule, depicted in Equations (25) and (26), and consequently, ROAs were made able to tackle core and subdissensions simultaneously by applying the modified OWGA operator (operator was able to act for qualitative modeling of only core level dimensions)

(vi) The authors applied dominance theory to conduct robust comparison amongst the CRPAI preferences, received by the three parts of the MUTIMOORA algorithm [40, 41].

(vii) The modified OWGA operator can be explored in future to tackle the extended or advanced CRP core-subdimensions

\section{Conclusions}

The coal is determined as a fossil fuel, which releases the several offensive monoparticles and high magnitude of $\mathrm{CO}_{2}$ to the atmosphere and also causes acid rain and high temperature ([6, 11-13]; CURC 2004; [1, 20]). It is well purported by the ecological scholars that CRP contributes in controlling the air pollution $[2,7,18,19,22,26,28,48,63]$. Therefore, to control such as air pollution and global warming vice versa, the presented research work enrolled a dynamic multidimension CRPAI structure accompanied with the ROA (modification of OWGAO appended with MULTIMOORA with application of dominance theory) algorithm to scrutinize and benchmark the best CRP from available options under linguistic information of expert's panel. This proposed research work assisted the managers to identify the best CRP practice and technique or to opt viable CRPs amongst preferred CRPs that will reduce the air pollution, consequently weaken the virus and ills, and make living people happy.

For making decision, manager can use the proposed dynamic multidimension CRPAI structure and invite the evaluated team of expert of own and other industries to view the preferred CRP alternatives one by one. Next, the expert would ask to provide the ratings against subdimensions of CRPAI structure by using proposed linguistic variable corresponding to IVFN. Next, assigned rating would be substituted by IVFN by using OWGOA. Later, the MUTIMOORA algorithm would deliver results for further comparison by dominance theory and making final decision. This proposed research work assists the managers of CRP to acquire the many coal supplying orders from coal user plants (which strictly follow the green policies). The proposed research dossier helps to finalize the best CRPAI technique or CRPs amongst available for winning the discussed twice factors such as coal logistic from coal refinery location to entry gate of coal user power plants and in process logistic/movement inside the power plants, linked to diminish the air pollution. The wok cannot shape other problems such as liner programming and route algorithm.

\section{Data Availability}

The data used to support the findings of this study are available in Tables 5-7.

\section{Conflicts of Interest}

The authors declare that there is no conflict of interest regarding the publication of this manuscript.

\section{References}

[1] Z. Bian, H. Wang, S. Mu, and H. Leng, "The impact of disposal and treatment of coal mining wastes on environment and farmland," in International Conference on Waste Management, Environmental Geotechnology and Global Sustainable Development (ICWMEGGSD'07-GzO'07), pp. 1-76, Ljubljana, Slovenia, 2007.

[2] P. Billing, "Emission of hazardous air pollutants from coal fired power plants," Environmental Health \& Engineering, vol. $5,2011$.

[3] Environmental compliance and performance report, Management of Dust from Coal Mines, Department of Environment and Climate Change and Water NSW, Sydney, 2010.

[4] F. S. Greb, C. F. Eble, D. C. Peters, and A. R. Papp, Coal and the Environment, AGI Environmental Awareness Series, 2006.

[5] B. G. Gottlieb, G. Steven, and L. G. Evans, Coal ash the toxic threat to our health and environment, Report from Physicians for Social Responsibility and Earthjustice, 2010.

[6] E. L. Heffern and D. A. Coates, "Geologic history of natural coal-bed fires, Powder River basin, USA," International Journal of Coal Geology, vol. 59, no. 1-2, pp. 25-47, 2004. 
[7] D. Katoria, D. Sehgal, and S. Kumar, "Environment impact assessment of coal mining," International Journal of Environmental Engineering and Management, vol. 4, pp. 245-250, 2013.

[8] V. Munnik, The Social and Environmental Consequences of Coal Mining in South Africa: A Case Study, Copyright: Environmental Monitoring Group, 2010.

[9] H. O. Rashid, S. Hossain, Z. Urbi, and S. Islam, "Environmental impact of coal mining: a case study on the Barapukuria coal mining industry," Bangladesh, Middle-East Journal of Scientific Research, vol. 21, no. 1, pp. 268-274, 2014.

[10] M. Shealy and J. P. Dorian, "Growing chinese coal use: dramatic resource and environmental implications," Energy Policy, vol. 38, no. 5, pp. 2116-2122, 2010.

[11] M. Keating, Radle to Grave: the environmental impacts from coal, Clean Air Task Force 77 Summer Street, Boston, MA, USA, 2001.

[12] T. E. Kjellstrom, A. Neller, and R. W. Simpson, "Air pollution and its health impacts: the changing panorama," Medical Journal of Australia, vol. 177, no. 11, pp. 604-608, 2002.

[13] P. J. Lloyd, Coal Mining and the Environment Energy Research Institute, University of Cape Town, Lloyd-Coal \& the Environment IBA Durban, 2002.

[14] K. L. Strellec, "Economic and Environmental Impact Assessment of Proposed Effluent Limitations Guidelines and Standards for the Coal Mining Industry," Remining and Western Alkaline Subcategories, vol. 3, 2000.

[15] J. I. Sams III and K. M. Beer, Effects of Coal-Mine Drainage on Stream Water Quality in the Allegheny and Monongahela River Basins-Sulfate Transport and Trends, National Water-Quality Assessment Program, 2000.

[16] D. Mamurekli, "Environmental impacts of coal mining and coal utilization in the UK," Acta Montanistica Slovaca Rocnik, vol. 15, pp. 134-144, 2010.

[17] L. Ruhl, A. Vengosh, G. S. Dwyer et al., "Survey of the potential environmental and health impacts in the immediate aftermath of the coal ash spill in Kingston, Tennessee," Environmental Science \& Technology, vol. 43, no. 16, pp. 6326-6333, 2009.

[18] D. Casper, R. Golob, M. Buchanan, J. Tobo, and J. Sykes, Guide to Prevention and Control of Infectious Diseases in the Workplace, A Joint Initiative BC service employees union and public service agency, 2007.

[19] S. P. Deshmukh and V. K. Sunnapwar, "Validation of performance measures for green supplier selection in Indian industries," International Journal of Modern Engineering Research (IJMER), vol. 3, no. 3, pp. 1617-1622, 2013.

[20] G. Langergraber and E. Muellegger, "Ecological Sanitation-a way to solve global sanitation problems?," Environment International, vol. 31, no. 3, pp. 433-444, 2005.

[21] V. Misra and S. D. Pandey, "Hazardous waste, impact on health and environment for development of better waste management strategies in future in India," Environment International, vol. 31, no. 3, pp. 417-431, 2005.

[22] M. K. Chien, "Influences of green supply chain management practices on organizational sustainable performance," International Journal of Environmental Monitoring and Protection, vol. 1, no. 1, pp. 12-23, 2014.

[23] G. T. Tsoulfas and C. P. Pappis, "A model for supply chains environmental performance analysis and decision making," Journal of Cleaner Production, vol. 16, no. 15, pp. 1647-1657, 2008.
[24] N. K. Sahu, A. K. Sahu, and A. K. Sahu, "Cluster approach integrating weighted geometric aggregation operator to appraise industrial robot," Kybernetes, vol. 47, no. 3, pp. 487-524, 2018.

[25] D. Styles, K. O'Brien, and M. B. Jones, "A quantitative integrated assessment of pollution prevention achieved by integrated pollution prevention control licensing," Environment International, vol. 35, no. 8, pp. 1177-1187, 2009.

[26] J. Annamalai and M. Namasivayam, "Endocrine disrupting chemicals in the atmosphere: Their effects on humans and wildlife," Environment International, vol. 76, pp. 78-97, 2015.

[27] S. M. Chen and J. H. Chen, "Fuzzy risk analysis based on ranking generalized fuzzy numbers with different heights and different spreads," Expert Systems with Applications, vol. 36, no. 3, pp. 6833-6842, 2009.

[28] A. Diabat and K. Govindan, "An analysis of the drivers affecting the implementation of green supply chain management," Resources, Conservation and Recycling, vol. 55, no. 6, pp. 659-667, 2011.

[29] X. X. Long and X. Jun, "Green supplier evaluation and controlling based on life cycle assessment theory'," Journal of Jiamusi University, Natural Science Edition, vol. 2, 2007.

[30] A. K. Sahu, N. K. Sahu, and A. Sahu, "Appraisal of CNC machine tool by integrated MULTI-MOORA-IGVN circumstances: an empirical study," in International Journal of Grey Systems: Theory and Application (IJGSTA), Emerald, Group Publishing limited, UK, 2014.

[31] C. Li, L. Xin, Z. Zhijie, D. Zhenghua, Y. Ji, and F. Wang, "Effect of heat treatment on structure and gasification reactivity of petroleum coke," International Journal of Coal Science \& Technology, vol. 3, pp. 53-61, 2016.

[32] R. Venugopal, J. P. Patel, and C. Bhar, "Coal washing scenario in India and future prospects," International Journal of Coal Science \& Technology, vol. 3, no. 2, pp. 191-197, 2016.

[33] H. Dhawan, S. Upadhyayula, and D. K. Sharma, "Design of experiments to optimize the extraction parameters of a power grade Indian coal," International Journal of Coal Science \& Technology, vol. 5, no. 4, pp. 417-429, 2018.

[34] F. Sitorus, J. J. C. Pablo, and R. B. Parada, "Multi-criteria decision making for the choice problem in mining and mineral processing: applications and trends," Expert Systems with Applications, vol. 121, no. 1, pp. 393-417, 2019.

[35] K. Pourabdollah, M. S. Zakaria, M. M. Najafizadeh Seyed, and F. Motaghedi, "Experimental investigation of process parameters during graphitization of catalytic coke," International Journal of Coal Science \& Technology, vol. 6, no. 4, pp. 611620, 2019.

[36] H. Dhawan, R. Kumar, S. Upadhyayula, K. K. Pant, and D. K. Sharma, "Fractionation of coal through organo-separative refining for enhancing its potential for the CO2-gasification," International Journal of Coal Science \& Technology, vol. 7, no. 3, pp. 504-515, 2020.

[37] A. Kumar Shukla, Z. Ahmad, M. Sharma et al., "Advances of carbon capture and storage in coal-based power generating units in an Indian context," Energies, vol. 13, no. 16, 2020.

[38] S. Chakraborty, "Applications of the MOORA method for decision making in manufacturing environment," International Journal Advanced Manufacturing Technology, vol. 54, no. 9-12, pp. 1155-1166, 2011.

[39] V. S. Gadakh, "Application of MOORA algorithm for parametric optimization of milling processes," International Journal of Applied Engineering Research, vol. 1, no. 4, 2011. 
[40] Z. Bu, M. Prabhu, R. R. Ahmed, and A. K. Sahu, "Exploiting GWmZd Model by Exploring Knowledge-Based GreyHolistic Technique for Sustainable Vendor Evaluation," Mathematical Problems in Engineering, vol. 2020, Article ID 8970947, 18 pages, 2020.

[41] Z. He, X. Ma, J. Luo, A. K. Sahu, A. K. Sahu, and N. K. Sahu, Exploitation of the Advanced Manufacturing Machine Tool Evaluation Model under Objective-Grey Information: A Knowledge-Based Cluster with the Grey Relational Analysis Approach, Grey Systems: Theory and Application, 2020.

[42] W. K. M. Brauers and E. K. Zavadskas, "Robustness of the MULTI-OBJECTIVE MOORA METHOD with a test for the facilities sector," Technological and Economic Development of Economy, vol. 15, no. 2, pp. 325-375, 2009.

[43] W. K. M. Brauers and E. K. Zavadskas, "Project management by MULTIMOORA as an instrument for transition economies / PROJEKTU VADYBA SU MULTIMOORA KAIP PRIEMONE PEREINAMOJO LAIKOTARPIO ÜKIAMS," Technological and Economic Development of Economy, vol. 16, no. 1, pp. 5-24, 2010.

[44] W. K. M. Brauers, A. Balezentis, and T. Balezentis, "MULTIMOORA for the EU member states updated with fuzzy number theory / NERAIŠKIŲJŲ SKAIČIU TEORIJA PAPILDYTAS MULTIMOORA METODAS EUROPOS SĄJUNGOS VALSTYBIŲ NARIŲ IŠSIVYSTYMO VERTINIMUI," Technological and Economic Development of Economy, vol. 17, no. 2, pp. 259-290, 2011.

[45] W. K. M. Brauers and E. K. Zavadskas, "MULTIMOORA optimization used to decide on a bank loan to buy property," Technologic al and Economic Development of Economy, vol. 17, no. 1, pp. 174-188, 2011.

[46] S. M. Chen and K. Sanguansat, "Analyzing fuzzy risk based on similarity measures between interval-valued fuzzy numbers," Expert Systems with Applications, vol. 38, no. 7, pp. 86128621, 2011.

[47] G. D. Li, D. Yamaguchi, and M. Nagai, “A grey-based decisionmaking approach to the supplier selection problem," Mathematical and Computer Modelling, vol. 46, no. 3-4, pp. 573581, 2007.

[48] M. Kutz, Environmentally conscious materials handling, John Wiley \& Sons, Inc., 2009.

[49] I. Linkov, F. K. Satterstrom, G. Kiker, C. Batchelor, T. Bridges, and E. Ferguson, "From comparative risk assessment to multicriteria decision analysis and adaptive management: Recent developments and applications," Environment International, vol. 32, no. 8, pp. 1072-1093, 2006.

[50] P. D. Liu, “A weighted aggregation operators multi-attribute group decision-making method based on interval-valued trapezoidal fuzzy numbers," Expert Systems with Applications, vol. 38, no. 1, pp. 1053-1060, 2011.

[51] P. D. Liu and F. Jin, “A multi-attribute group decision-making method based on weighted geometric aggregation operators of interval-valued trapezoidal fuzzy numbers," Applied Mathematical Modelling, vol. 36, no. 6, pp. 2498-2509, 2012.

[52] R. R. Yager, "On ordered weighted averaging aggregation operators in multicriteria decisionmaking," IEEE Transactions on Systems, Man and Cybernetics, vol. 18, no. 1, pp. 183-190, 1988.

[53] P. D. Liu, "An extended TOPSIS algorithm for multiple attribute group decision making based on generalized intervalvalued trapezoidal fuzzy numbers," Informatica, vol. 35, pp. 185-196, 2011.
[54] J. Zhang, D. Wu, and D. L. Olson, "The method of grey related analysis to multiple attribute decision making problems with interval numbers," Mathematical and Computer Modelling, vol. 42, no. 9-10, pp. 991-998, 2005.

[55] S. H. Chen, "Operations of fuzzy numbers with step form membership function using function principle," Information Sciences, vol. 108, no. 1-4, pp. 149-155, 1998.

[56] T. H. Comes, M. Hiete, N. Wijngaards, and F. Schultmann, "Decision maps: A framework for multi-criteria decision support under severe uncertainty," Decision Support Systems, vol. 52, no. 1, pp. 108-118, 2011.

[57] S. H. Wei and S. M. Chen, "Fuzzy risk analysis based on interval-valued fuzzy numbers," Expert Systems with Applications, vol. 36, no. 2, pp. 2285-2299, 2009.

[58] H. J. Zimmermann, Fuzzy Set Theory and Its Applications, Kluwer Academic Publishers, Boston, Dordrecht, London, Second Eds edition, 1991.

[59] A. Zakarian, "Analysis of process models: a fuzzy Logic approach," International Journal of Advanced Manufacturing Technology, vol. 17, no. 6, pp. 444-452, 2001.

[60] L. A. Zadeh, "Fuzzy sets," Information and Control, vol. 8, no. 3, pp. 338-353, 1965.

[61] L. A. Zadeh, "The concept of a linguistic variable and its application to approximate reasoning-I," Information Sciences, vol. 8, no. 3, pp. 199-249, 1975.

[62] T. Balezentis and S. Zeng, "Group multi-criteria decision making based upon interval-valued fuzzy numbers: An extension of the MULTIMOORA method," Expert Systems with Applications, vol. 40, no. 2, pp. 543-550, 2013.

[63] P. K. Dey and W. Cheffi, "Green supply chain performance measurement using the analytic hierarchy process: a comparative analysis of manufacturing organisations," Production Planning \& Control: The Management of operations, vol. 24, no. 8-9, pp. 702-720, 2013. 